\title{
Activités
}

$14-2$ | 2017

Dimensions et conditions d'une intervention capacitante

\section{Co-construire l'autopoïèse organisationnelle : le Laboratoire Développemental comme modèle et comme moyen de l'intervention capacitante}

Co-constructing an autopoietic organization: the Developmental Laboratory as a model and means of the Enabling Intervention

\section{Gianna Carta et Pierre Falzon}

\section{(2penEdition}

Journals

Édition électronique

URL : http://journals.openedition.org/activites/3022

DOI : $10.4000 /$ activites.3022

ISSN : 1765-2723

Éditeur

ARPACT - Association Recherches et Pratiques sur les ACTivités

\section{Référence électronique}

Gianna Carta et Pierre Falzon, « Co-construire l'autopoï̀se organisationnelle : le Laboratoire Développemental comme modèle et comme moyen de l'intervention capacitante », Activités [En ligne], 14-2 | 2017, mis en ligne le 15 octobre 2017, consulté le 20 avril 2019. URL : http:// journals.openedition.org/activites/3022 ; DOI : 10.4000/activites.3022

Ce document a été généré automatiquement le 20 avril 2019

\section{c) (i) $\Theta$}

Activités est mis à disposition selon les termes de la licence Creative Commons Attribution - Pas d'Utilisation Commerciale - Pas de Modification 4.0 International. 


\section{Co-construire l'autopoïèse organisationnelle : le Laboratoire Développemental comme modèle et comme moyen de l'intervention capacitante}

Co-constructing an autopoietic organization: the Developmental Laboratory as a model and means of the Enabling Intervention

Gianna Carta et Pierre Falzon

\section{NOTE DE L'ÉDITEUR}

Article soumis le 31/07/2016, accepté le 25/07/2017

1 Sur la base d'une analyse réflexive a posteriori d'une intervention ergonomique, cet article vise à présenter les conditions de mise en œuvre et les facteurs de réussite d'une intervention capacitante en accompagnement de la transformation organisationnelle. Par intervention capacitante, nous entendons une intervention qui, dans un objectif de changement, s'appuie sur une démarche de développement et vise sa pérennisation.

Dans un premier temps, nous présenterons la perspective de l'organisation dans laquelle la recherche s'inscrit, ainsi que le modèle qu'inspire l'intervention ergonomique et les dispositifs mis en place.

Ensuite, on décrira les éléments clés de l'intervention et les productions stratégiques, c'est-à-dire les choix d'agencement de l'environnement et les actions mises en place par l'ergonome visant le développement et la durabilité de l'action engagée. Enfin, les principales contributions et ouvertures pour la formation initiale et pour la pratique d'intervention seront discutées. 


\section{Contexte : un projet de reconception des processus organisationnels d'une entité de maintenance de la signalisation métropolitaine}

4 L'intervention présentée ici a été réalisée au sein de l'entité en charge de la maintenance des dispositifs de signalisation du métro parisien. Pour répondre à des enjeux économiques de réduction des coûts de la maintenance et de compétitivité sur le marché mondial, l'entreprise a réalisé une internalisation partielle de cette activité auparavant entièrement sous-traitée au constructeur de ces dispositifs de signalisation. Un ensemble de transformations ont été réalisées de manière top-down, portant essentiellement sur les aspects structurels. L'entité de maintenance comprend cinq fonctions (logistique, ingénierie de maintenance, travaux sur le réseau, analyse de la performance et centres opérationnels). À l'origine, ces fonctions étaient spécialisées par type de système de signalisation et dispersées géographiquement. Suite au processus d'internalisation, chaque fonction a pris en charge l'ensemble des systèmes de signalisation, et les cinq fonctions ont été regroupées dans un même bâtiment. Les conséquences de cette nouvelle configuration concernent d'une part la performance globale de l'entité de maintenance, d'autre part et surtout la fiabilité et la résilience de l'organisation même.

5 L'intervention ergonomique a été déclenchée à ce moment de la vie organisationnelle afin d'analyser le système de maintenance et de caractériser les conséquences de ces changements sur le fonctionnement de l'entité. Une analyse de l'activité de dépannage, des interfaces entre les agents de maintenance et les fonctions support et des interfaces entre l'entité de maintenance et les autres acteurs de la conception (concepteurs, réalisateurs et exploitants) a été réalisée.

6 L'analyse du travail a mis en évidence un fonctionnement organisationnel caractérisé par : 1) des objectifs, des moyens, des langages, et une action qui restent cloisonnés par fonction (action en silo) ; 2) une connaissance limitée du travail des autres fonctions en termes d'objectifs poursuivis, d'opérations effectuées, de modalités de travail, des délais nécessaires à chacun ; 3) des pratiques non partagées et parfois contradictoires entre les différentes fonctions; 4) l'absence d'une vision systémique partagée entre les fonctions en ce qui concerne le rôle et les apports de chacune dans le processus global de maintenance et le rôle de l'entité dans le cycle de vie des projets de réalisation des systèmes de signalisation. Ces contradictions organisationnelles freinent l'activité de chaque fonction et entravent la continuité et la fiabilité du service global fourni par l'entité. Les agents de chaque fonction sont amenés à mettre en place des stratégies de régulation, elles aussi cloisonnées par fonction, qui sont souvent coûteuses en terme de temps et de ressources.

7 Ces difficultés semblent avoir pour origine un modèle managérial focalisé sur la dimension technique et négligeant la dimension socio-organisationnelle.

8 Suite à la mise en évidence de ces blocages organisationnels, nous avons proposé un projet de reconception participative des processus organisationnels. Ce projet visait à accompagner les acteurs dans la conception de solutions organisationnelles (process, organisation du travail, procédures, etc.) qui remettent au centre le travail, en tant que facteur clé pour le développement des collectifs et de l'organisation. Ce projet avait pour ambition de construire les compétences des acteurs impliqués au cours même de la 
transformation organisationnelle, de manière qu'ils puissent par la suite réaliser un renouvellement continu de leur organisation, au-delà du dispositif d'intervention de l'ergonome.

9 On présentera en section 2 le cadrage théorique qui sous-tend les choix de structuration et de conduite de l'intervention. On décrira notamment la vision processuelle de l'organisation et le concept d'autopoïèse, qui fondent la volonté développementale et pérenne du dispositif d'intervention.

\section{Une vision processuelle d'une organisation autopoïétique}

\subsection{Une vision processuelle}

10 L'intervention adopte une vision processuelle de l'organisation. Dans cette perspective, les organisations ne sont pas vues comme des objets statiques (structures), mais comme des processus dynamiques en reconstruction permanente via de ses acteurs (Feldman, 2000 ; Hernes, 2008, 2010 ; Hernes \& Maitlis, 2010 ; Tsoukas, 2009 ; Tsoukas \& Chia, 2002 ; Weick, 2012). Karl Weick, un des contributeurs majeurs de cette perspective, souligne ce caractère dynamique et émergent de l'organisation et de son fonctionnement et, pour la qualifier, choisit un verbe d'action - organizing - plutôt que l'habituel substantif organization.

11 L'organisation est donc vue comme un processus organisant (Lorino, 2013) qui émerge d'un travail continu et collectif de sensemaking. Ce dernier est un moyen collectif pour faire face à l'incertitude, aux discordances et à l'ambiguïté des situations de travail vécues (Weick, Sutcliffe, \& Obstfeld, 2005). Les discordances peuvent concerner un écart entre ce qui est demandé et ce qui est à disposition pour le faire, écart pris en charge par les opérateurs, avec des conséquences plus ou moins capacitantes. Mais elles peuvent aussi concerner les hiatus entre mondes professionnels hétérogènes, aux représentations, objectifs, langages, repères, pratiques différents, parfois éloignés spatialement et temporellement, qui sont appelés à travailler ensemble dans le but d'aboutir à une production commune. Le caractère indéterminé des situations peut être lié également à un non-accompagnement des évolutions organisationnelles qui modifient les modalités habituelles de travail. Ces situations, si elles ne sont pas outillées de façon efficace pour permettre un agir transverse, peuvent amener à une faillite du sens pour les acteurs concernés, avec des conséquences négatives en termes de santé, de sécurité et de performance.

Le processus de sensemaking renvoie donc à une dynamique dialogique à travers laquelle les acteurs organisationnels font évoluer de manière continue le sens (le pré-réfléchi) et la signification (le réfléchi) de leurs actions sur et dans l'environnement (Weick, 1995). Ainsi, le sensemaking pourrait être lu comme la mise en intrigue (Ricœur, 1984) de la situation en question. C'est une recherche collective de l'intelligibilité d'une situation à travers la narration d'une histoire dont les acteurs concernés connaissant déjà la fin. Cette dernière permet aux acteurs de reconstruire les enchainements des événements et les liens de cause à effets et de mettre un ordre plausible à des évènements plus ou moins désordonnés et ambigus afin de rendre l'action intelligible. 
13 À partir de la vision weickienne et de la perspective processuelle dans laquelle il s'inscrit, on peut tirer deux conséquences pour la théorie de l'organisation et l'action sur celle-ci.

Premièrement, les pratiques ne sont pas séparées de l'organisation, mais en constituent l'essence (Gherardi, 2000 ; Nicolini, Gherardi \& Yanow, 2003 ; Orlikowski, 2000, 2007). Le niveau d'analyse se déplace ainsi des formes organisationnelles et des modes de structuration (statiques) vers les processus (dynamiques), c'est-à-dire vers la façon par laquelle les organisations émergent à partir des dynamiques collectives quotidiennes. Il y a une totale équivalence entre sensemaking et organizing (Weick, 1995). L' organizing correspond à un effort collectif et permanent de transformation du monde au moment même où on en reconstruit le sens (Lorino, Tricard, \& Clot, 2011). En ce sens l'organisation émerge de l'action transverse elle-même : «Il n'y pas d'activité collective sans organisation, et pas de processus organisant per se, indépendamment d'une action à accomplir » (Lorino, 2013, p. 222). La représentation de l'expérience se construit dans l'action et le sens de ce qui est représenté forme la base pour l'action suivante (Hermes, 2007).

Deuxièmement, l'organisation-structure comme un état éphémère, temporellement situé, traversé par des processus de self-design permanents (Feldman, 2000 ; Weick, 1977) amène à une vision particulière du changement organisationnel. Les organisations-structures sont des "arrêts temporaires » dans une mer de flux et de transformations continues (Chia 2002). Cependant, le fait que le changement soit une caractéristique endémique et constitutive de l'organisation ne signifie pas que les transformations réalisées soient également capacitantes pour l'ensemble des acteurs et de leurs activités (Falzon, 2005). En termes d'analyse et d'intervention organisationnelle, ceci implique tout d'abord qu'étudier le changement ne consiste pas à décrire le passage d'un état $\mathrm{A}$ à un état $\mathrm{B}$, comme un processus planifiable et complètement contrôlable. Ceci implique aussi de se focaliser davantage sur comment les micro-transformations sont co-construites dans les pratiques au cours de leur évolution (Tsoukas \& Chia, 2002) et comment leur influence se répand et interagit avec le contexte spécifique. Ceci implique enfin d'outiller et d'accompagner les acteurs organisationnels dans la transformation capacitante de leurs pratiques à travers la mise en place d'un environnement développemental à visée durable.

\subsection{Vers l'autopoïèse organisationnelle}

Pour répondre à cet enjeu de développement à visée durable, la méthode d'action a été conçue en s'inspirant de la notion d'autopoïèse. Le terme dérive du grec ancien et compose les mots «autos", c'est-à-dire soi, et "poiein » qui renvoie à créer, produire (Quick, 2003). Il trouve son origine dans le champ de la neurobiologie. La théorie de l'autopoï̀se (Maturana \& Varela, 1980; Varela, Maturana, \& Uribea, 1974) définit l'organisation du vivant comme l'expression d'un processus autoreproducteur. Selon Varela : « un système autopoḯtique est organisé comme un réseau de processus de production de composants qui (a) régénèrent continuellement par leurs transformations et leurs interactions le réseau qui les a produits, et qui (b) constituent le système en tant qu'unité concrète dans l'espace 
où il existe, en spécifiant le domaine topologique où il se réalise comme réseau " (Varela, 1989, p. 45).

Le concept d'autopoïèse a été appliqué à l'approche de la transformation des processus de l'entité de maintenance. Pour que cette dernière puisse se définir en tant que système autopoḯtique, l'entité doit être capable :

- de définir ses composantes, ses processus de production, ses règles de fonctionnement et d'identifier ses besoins et ses points de blocage ;

- de développer une réflexivité sur ses propres pratiques de gestion et d'autorégulation et d'en définir les critères. Cette réflexivité renvoie à la façon par laquelle le système apprend à apprendre;

- pour pouvoir mettre en mouvement et ancrer les mécanismes autopoiétiques dans la culture organisationnelle, de co-construire et de formaliser les conditions de sa viabilité.

Afin d'accompagner les acteurs organisationnels dans ce travail de co-construction et de mise en marche de mécanismes autopoiétiques, l'intervention a été pensée et outillée comme un processus formatif. On appellera «Laboratoire Développemental » (LD) l'ensemble du dispositif d'intervention et $d$ 'apprentissage, ces deux processus apparaissant comme indissociables. Le Laboratoire Développemental, détaillé en section 3, prend la dimension constructive comme objet premier de l'intervention afin de répondre aux objectifs développementaux. La contribution de ce travail à la recherche sur l'intervention capacitante sera soulignée. Ensuite, les composantes formelles, les objectifs et hypothèses sous-jacentes et les productions réalisées seront présentés.

\section{Une intervention à visée développementale pérenne}

Cette intervention s'inscrit dans la perspective de l'ergonomie constructive (Falzon, 2013). Celle-ci défend une vision de l'homme comme autonome, créateur de solutions et auteur de sa propre transformation. Le développement des individus, des collectifs et des organisations, est au cœur de cette perspective. Intervenir dans une visée développementale signifie créer des conditions d'action qui favorisent la construction des ressources nécessaires à la réussite. Ceci demande la construction et la systématisation d'espaces de débat réflexif sur les pratiques (Casse, 2015; Rocha, Mollo, \& Daniellou, 2015), l'élargissement des marges de manœuvre (de décision et d'action) des acteurs permettant le renouvellement durable de règles effectives. Construire les conditions de réussite signifie également faciliter la construction de savoir-faire, de connaissances, de compétences. L'intervention développementale vise à favoriser les apprentissages au cours même de son déroulement (Falzon \& Barcellini, 2014), et à promouvoir la conception de systèmes de travail qui eux-mêmes favoriseront les apprentissages et le développement (Arnoud \& Falzon, 2017 ; Barcellini, 2015). En ce sens, certains auteurs ont interprété l'intervention comme un acte pédagogique (Dugué, Petit, \& Daniellou, 2010; Noyer \& Barcellini, 2014), au cours duquel l'ergonome mobilise les registres de l'expertise, de l'apprentissage et de la relation pour rendre les individus capables en situation et pour faciliter le développement personnel. Le développement des activités et des situations de travail est vu comme un « effet positif induit » (Barcellini, Van Belleghem, \& Daniellou, 2013). Le fait que les acteurs apprennent est une conséquence de la mise en place de structures participatives capacitantes (Petit, 2005 ; Raspaud, 2014) ; mais peu d'études ont pris la dimension constructive comme visée/objet même de l'action. L'intervention présentée ici veut représenter une première contribution dans cette direction. En 
particulier, la méthodologie proposée vise la durabilité des effets capacitants à travers la construction et la mise en marche de mécanismes autopoïétiques (Carta, Falzon, \& Re, 2015), qui permettent à l'organisation de se renouveler de manière autonome selon les besoins émergents de l'activité et de son environnement.

\subsection{Les composantes formelles du « Laboratoire développemental »}

La méthode d'action proposée dans cette intervention correspond à la conduite d'un projet d'accompagnement à la transformation organisationnelle (Engeström, Sannino, Virkkunen, 2014 ; Petit, 2005, 2006 ; Raspaud, 2014 ; Seim, Broberg, \& Andersen, 2014 ; StVincent, Vézina, Bellemare, Denys, Ledoux, \& Imbeau, 2011).

Ce projet a débuté en avril 2011 et s'est achevé à la fin d'août 2015. La chronique des différentes phases et les finalités de chacune sont synthétisées dans le tableau 1.

La méthode d'action proposée dans l'intervention articule des techniques de diagnostic du travail et des instances chargées des arbitrages décisionnels (COPIL), du lancement et du suivi (réunions) et de la production de connaissances sur l'organisation actuelle et future (groupes de travail). L'ensemble des dispositifs mis en place dans les différentes phases de la démarche est présenté dans la figure 1.

Tableau 1 : Phases et finalités des dispositifs d'intervention. Table 1: Steps and purposes of the intervention devices

\begin{tabular}{|c|c|c|c|}
\hline Délai & $\begin{array}{l}\text { Dénomination } \\
\text { de la phase }\end{array}$ & Action ergonomique & Finalité \\
\hline $\begin{array}{l}04 / 2011 \\
\text { à } \\
05 / 2013\end{array}$ & Pré-implantation & $\begin{array}{l}\text { Diagnostic ergonomique } \\
\mathrm{du} \text { système de } \\
\text { maintenance }\end{array}$ & $\begin{array}{l}\text { - Fonder stratégiquement } \\
\text { politiquement le projet. } \\
\text { - Instruire les intentions de } \\
\text { transformation et orienter les } \\
\text { décisions d'action. } \\
\text { - Identifier les }\end{array}$ \\
\hline $\begin{array}{l}10 / 2013 \\
\text { à } \\
05 / 2014\end{array}$ & $\begin{array}{l}\text { Travail } \\
\text { d'organisation } \\
\text { transverse }\end{array}$ & $\begin{array}{l}\text { Conduite d'une démarche } \\
\text { participative de } \\
\text { reconception } \\
\text { processus } \\
\text { organisationnels }\end{array}$ & $\begin{array}{l}\text { - Définir le système à travers la co- } \\
\text { conception et le test de nouvelles } \\
\text { solutions organisationnelles } \\
\text { (processus, outils, méthodes, formes } \\
\text { de coordination et de management, } \\
\text { etc.) }\end{array}$ \\
\hline
\end{tabular}




\begin{tabular}{|c|c|c|c|}
\hline $\begin{array}{l}05 / 2014 \\
\text { à } \\
08 / 2015\end{array}$ & $\begin{array}{l}\text { Ancrage des } \\
\text { mécanismes } \\
\text { autopoḯtiques }\end{array}$ & $\begin{array}{lr}\text { Transfert de la conduite } \\
\text { d'une démarche } \\
\text { participativer de } \\
\text { conception } \\
\text { nouvelle prescription }\end{array}$ & $\begin{array}{l}\text { - Réfléchir au fonctionnement du } \\
\text { système à travers la réalisation d'un } \\
\text { retour d'expérience sur les pratiques } \\
\text { de conduite d'une transformation } \\
\text { organisationnelle. } \\
\text { - Co-construire et formaliser la } \\
\text { viabilité du système à travers la co- } \\
\text { conception et la formalisation de } \\
\text { nouvelles prescriptions concernant la } \\
\text { production, la coordination et le débat } \\
\text { sur le travail. }\end{array}$ \\
\hline
\end{tabular}

Le diagnostic initial a fourni les éléments concernant à la fois les besoins d'apprentissage à satisfaire et les zones critiques du processus global de maintenance que les acteurs organisationnels devaient analyser et reconcevoir. Les connaissances ainsi construites sur le processus et sur ses acteurs ont permis à l'ergonome de formuler les exigences pour la conception de l'architecture du Laboratoire développemental (LD, qui sera décrit plus précisément dans la sous-section suivante). Le diagnostic initial a fourni le «matériel brut» permettant de construire la suite de l'intervention. Le Laboratoire Développemental s'intéresse aux phases de transformation pérenne de l'organisation, notamment celle $d u$ "Travail d'organisation transverse» et celle "d'Ancrage des mécanismes autopoḯtiques ». Le Laboratoire Développemental est structuré de manière à mettre en œuvre une volonté autopoḯtique de transformation de l'organisation. Ainsi, le LD représente un environnement exemplaire où les acteurs mobilisés expérimentent, c'est-à-dire font expérience et testent, une nouvelle manière (à visée durable) de construire leur organisation.

Figure 1 : Phasage et composantes formelles de l'intervention. Figure 1: Steps and formal components of the intervention

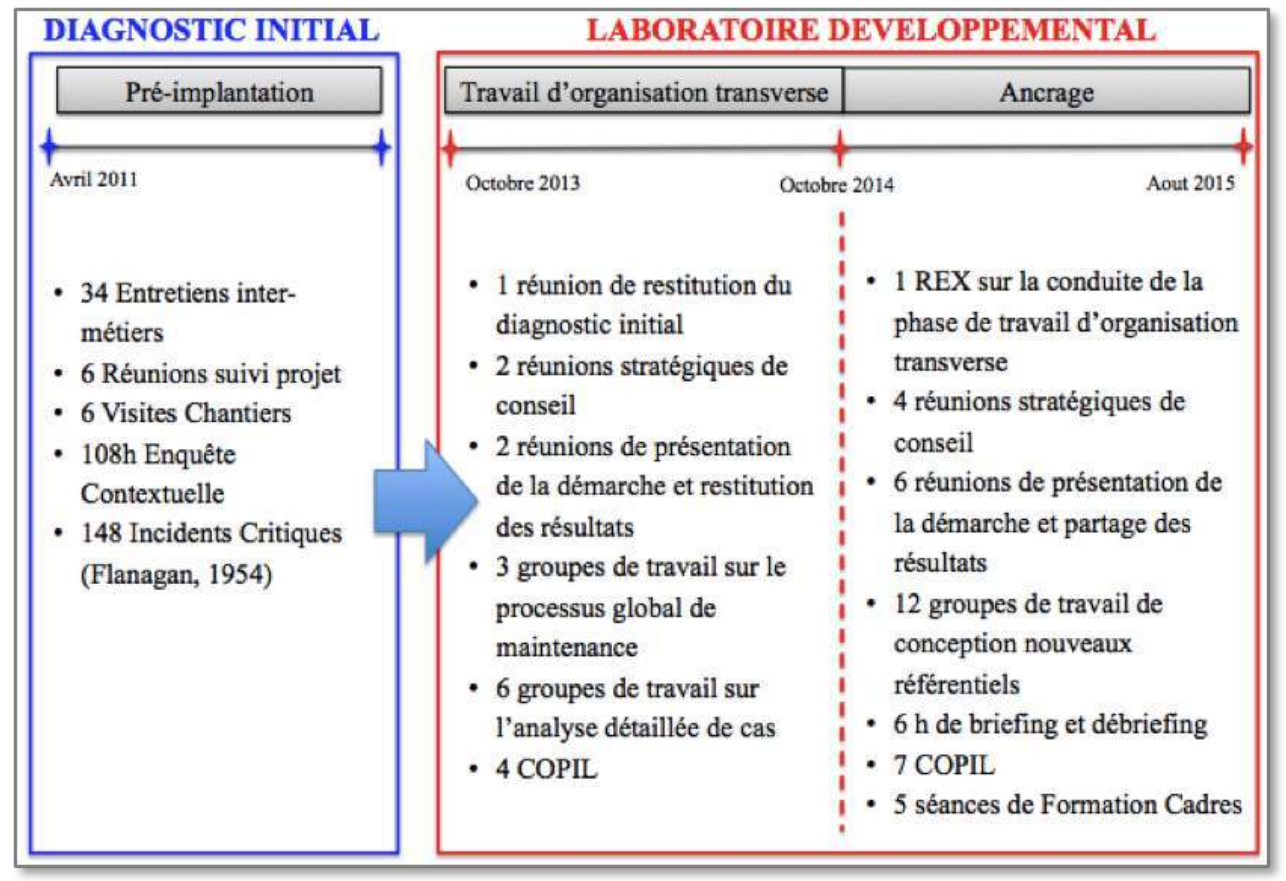



visée pérenne en accompagnement de la transformation organisationnelle : le texte se concentrera donc sur la présentation du LD et les logiques de fonctionnement sousjacentes.

positifs s'inscrivant dans le LD ont mobilisé 44 managers la maintenance de la signalisation et appartenant à 5 strates hiérarchiques (cf. Tableau 2). Dans la visée d'une professionnalisation de la fonction managériale, tout l'encadrement de proximité, les managers de fonction et de direction au niveau local et les managers $\mathrm{RH}$ et QHSE et de direction au niveau central ont participé activement aux groupes de travail inscrits dans le LD. Néanmoins, la mise à l'épreuve des solutions organisationnelles produites dans ces dispositifs a concerné l'ensemble de l'effectif de l'entité de maintenance, soit un total d'environ 120 agents.

Tableau 2 : Niveaux hiérarchiques des participants au Laboratoire Développemental. Table 2: Hierarchical levels of the Developmental Laboratory's stakeholders hierarchical

\begin{tabular}{|l|l|}
\hline \multicolumn{1}{|c|}{ Niveau Local } & \multicolumn{1}{c|}{ Niveau central } \\
\hline 1 manager de direction & 1 manager de direction \\
\hline 6 managers de fonction & 3 managers de fonction \\
\hline 33 managers de proximité & \\
\hline
\end{tabular}

\subsection{La mise en place d'un « Laboratoire développemental » : l'apprentissage comme condition et moyen}

La démarche s'inscrit dans le paradigme épistémologique socioconstructiviste (Dewey, 1938 ; Vygotsky, 1930/1983), qui met en avant le caractère social, situé et dialogique de la construction des savoirs, où l'environnement a un rôle de médiateur des apprentissages. L'apprentissage des acteurs est interprété comme un moyen et une condition pour le développement dont la systématisation s'est traduite par la mise en place d'un «Laboratoire Développemental» (LD) de l'organisation et de ses acteurs. L'ingénierie dispositifs du LD vise à faciliter le développement des apprentissages nécessaires, en faisant faire l'expérience et en permettant la mise à l'épreuve de nouvelles manières d'organiser les pratiques au cours même de ces productions. Le fonctionnement du LD se fonde sur deux concepts.

En premier lieu sur celui de «zone proximale de développement» de Vygotsky (1997) entendue ici comme la distance entre l'état actuel de l'entité, défini lors du diagnostic ergonomique, et l'état potentiel auquel elle peut arriver, que l'on peut déterminer empiriquement en examinant comment les acteurs organisationnels parviennent à résoudre les problèmes dans le cadre de l'environnement mis en place par l'ergonome. Cet environnement, qui inclut l'activité de l'ergonome lui-même, joue le rôle d'un facteur de conversion positif, comme Raspaud (2014) le propose, reprenant la terminologie d'Amartya Sen.

En second lieu sur celui de « relation de tutelle » (Bruner, 1983) qui renvoie aux stratégies mises en place par l'ergonome pour amener les acteurs organisationnels à construire les 
ressources et les solutions nécessaires au développement de l'organisation. En ce sens l'ergonome réalise des actions de simplification de la complexité de la tâche, d'aide à la résolution, de support à l'engagement et selon le principe de desétayage (Bruner, 1983) il prive progressivement les participants de son aide pour leur permettre de réaliser en autonomie la tâche.

L'ingénierie du LD présente les caractéristiques suivantes :

- la mise en place de situations de résolution de problèmes (issus de l'activité de maintenance) permettant de développer les compétences et les nouveaux concepts souhaités. Le séquencement est pensé de manière à faciliter l'appropriation des différentes situations (Mayen, 2012), à travers l'instauration de possibilités d'interaction avec l'environnement professionnel ou social ;

- la mise en marche d'une activité conjointe (Lorino, 2009) des différents niveaux hiérarchiques des fonctions et des services de l'entreprise ;

- l'utilisation d'objets frontières (Broberg, Andersen, \& Seim, 2011) permettant de rendre visible le travail de chacun et de déclencher un travail d'enquête (Dewey, 1938; Lorino \& Mourey, 2013) réflexive (Pastré, 2005 ; Schön, 1983) pour que les acteurs puissent renégocier les sens des situations (Weick, 2012) par la confrontation de différentes logiques d'action.

Dans ce travail de systématisation de la dimension constructive, l'ergonome est à la fois médiateur, garant et promoteur des conditions d'apprentissage et des apprentissages mêmes, comme on le verra plus loin

\subsection{Les objectifs et les hypothèses initiaux de l'intervention}

31 La phase de diagnostic ergonomique initiale réalisée par l'ergonome a permis de mettre en évidence les éléments de développement potentiel et de fournir ainsi l'orientation initiale de la démarche de reconception participative des processus organisationnels. En particulier, le défi de l'intervention n'était pas seulement d'outiller les acteurs organisationnels pour une co-conception de solutions organisationnelles plus capacitantes. Dans une visée développementale, l'objectif primaire était aussi de faire en sorte que ces mêmes acteurs soient capables de reconstruire leur organisation de manière autonome et durable sur la base des besoins émergents de l'activité.

Pour répondre à ce défi, l'action ergonomique a été pensée et agencée de manière à anticiper le départ de l'ergonome. Ceci s'est traduit par la systématisation, depuis les premières phases de l'intervention, du transfert vers les acteurs des compétences en reconception transverse et durable de l'organisation. Pour ce faire, l'ingénierie de l'intervention a accompagné et outillé les dimensions productives et constructives de l'activité (Samurçay \& Rabardel, 2004) à travers lesquels les acteurs organisationnels transforment à la fois le contexte et eux-mêmes. Ainsi, trois types d'objectifs ont été poursuivis et enrichis tout au long de la démarche : des objectifs productifs, constructifs et autopoḯtiques visant la durabilité des deux premiers.

Les objectifs productifs de l'intervention concernent à la fois des solutions organisationnelles plus capacitantes (résultat) et des manières de travailler ensemble (processus), elles aussi plus capacitantes, qui permettent de produire et de renouveler les solutions.

L'enjeu pour le dispositif d'intervention est d'outiller une activité collaborative de conception de nouvelles pratiques. Ceci demande aux acteurs de redéfinir les processus 
nécessaires à l'accomplissement des tâches, processus auparavant non définis, ou défaillants, ou organisés en silos. Par exemple, comment s'y prendre pour résoudre un incident complexe impactant la disponibilité ferroviaire, qui nécessite l'intervention dispersée temporellement et géographiquement de collectifs différents? La solution organisationnelle implique que les acteurs s'accordent sur une définition commune de ce qu'est un incident complexe, des éléments déclencheurs de ce type d'incident, des objectifs collectifs à poursuivre, des étapes clés qui structurent le processus de résolution et de la répartition des rôles et des responsabilités. Cela signifie également de concevoir les supports de travail adaptés aux besoins respectifs de chacun. Par exemple, les acteurs sont appelés à concevoir le dossier de traitement de l'incident, dossier qui constitue un objet frontière entre différents métiers. Ceci amène à définir de manière partagée le contenu (fiche technique, niveau d'analyse des expertises techniques, check-lists de dépannage), le format et les parties prenantes impliquées et les règles de clôture du dossier, tout en prenant en compte la variabilité et la diversité de l'activité transverse. Dans une optique de renouvellement durable des pratiques, les objectifs productifs renvoient également à la co-conception et l'intégration dans la culture organisationnelle des démarches participatives de retour d'expérience axée sur les processus organisationnels et des supports de travail, telles que les grilles REX ou les procédures correspondantes.

Les objectifs constructifs de l'intervention concernent le développement des compétences collectives nécessaires aux acteurs organisationnels pour réaliser et renouveler les solutions organisationnelles attendues (résultat et processus). L'apprentissage est vu ici comme condition et comme moyen du développement des collectifs et de l'organisation. Ainsi, face aux constats de faillite du collectif transverse, d'une activité cloisonnée par fonction et de la nécessité de produire des processus organisationnels transverses, l'enjeu constructif pour l'intervention est de développer une compétence collective (Boreham, 2004). Pour ce faire les dispositifs d'intervention visent à développer trois types de savoirs.

Le premier savoir concerne un sens partagé des évènements. Cela signifie aider les acteurs organisationnels à construire un vocabulaire commun et des modalités communes permettant de définir des zones et des processus techniques. Autrement dit, construire une référence partagée par les différents métiers concernés.

Le deuxième savoir renvoie à une conscience de l'interdépendance entre les différents acteurs organisationnels devant interagir. Cela demande de savoir apprécier et reconnaître la diversité des représentations, des identités, des besoins et des contraintes de chacun et les impacts sur les activités respectives afin de savoir négocier une activité conjointe. Ceci permet aux acteurs de se coordonner, de répondre de manière synergique et de donner de la valeur aux compétences spécifiques de chacun.

d'analyser l'organisation de manière holistique et systémique (Wilson, 2012).

Les objectifs autopoïétiques renvoient à la volonté de promouvoir et de faciliter l'ancrage dans la culture organisationnelle de ce travail d'organisation capacitant et des compétences collectives relatives. L'enjeu est de construire progressivement la mémoire des actions réalisées au cours de l'intervention. Ceci demande de pouvoir garder des traces des solutions construites (procédures, méthodes, espaces de débat sur le travail) permettant par la suite de continuer à renouveler le questionnement sur l'organisation. 


\subsection{Productions matérielles, productions immatérielles : construire un changement durable}

\section{aux moyens déployés à chaque étape.}

Tableau 3 : Récapitulatif des principaux résultats de l'intervention. Table 3: Summary of the main results of the intervention

\begin{tabular}{|c|c|c|c|c|}
\hline Etape & $\begin{array}{l}\text { Productions } \\
\text { stratégiques }\end{array}$ & Moyens & $\begin{array}{l}\text { Productions immatérielles } \\
\text { (dimension constructive) }\end{array}$ & $\begin{array}{l}\text { Productions matérielles } \\
\text { (dimension productive) }\end{array}$ \\
\hline $\begin{array}{l}1 \\
\text { Construire une } \\
\text { vision globale } \\
\text { et systémique }\end{array}$ & $\begin{array}{l}\text { - Diagnostic } \\
\text { - Cahier des charges } \\
\text { - Cartographie du } \\
\text { processus global } \\
\text { - Forme de democratie } \\
\text { exemplaire }\end{array}$ & $\begin{array}{l}\text { Co- } \\
\text { construction } \\
\text { du diagnostic } \\
\text { global }\end{array}$ & $\begin{array}{l}\text { - ROC } \\
\text { - Intelligibilité mutuelle } \\
\text { - Vision systeme } \\
\text { - Compétence analytique } \\
\text { - Engagement }\end{array}$ & $\begin{array}{l}\text { ¿Situations d'interface } \\
\text { saillantes " : cas à } \\
\text { mobiliser pour l'analyse } \\
\text { détaillèe }\end{array}$ \\
\hline $\begin{array}{c}2 \\
\text { Intervenir de } \\
\text { manière } \\
\text { transverse }\end{array}$ & $\begin{array}{l}\text { - Effacement graduel } \\
\text { de l'ergonome } \\
\text { - Fiches/cosignes }\end{array}$ & $\begin{array}{l}\text { Analyses } \\
\text { détailles et } \\
\text { production de } \\
\text { solutions } \\
\text { organisationn } \\
\text { elles }\end{array}$ & $\begin{array}{l}\text { - Compétence d'auto-enquête } \\
\text { - Montée de la prise } \\
\text { d'initiative des acteurs } \\
\text { - Compétence de résolution } \\
\text { de problèmes (co-design) } \\
\text { - Vision partagée de } \\
\text { l'organisation comme objet } \\
\text { manipulable }\end{array}$ & $\begin{array}{l}\text { Cartographies détaillées } \\
\text { Plan d'actions }\end{array}$ \\
\hline $\begin{array}{c}3 \\
\text { Réfléchir le } \\
\text { fonctionnement } \\
\text { du système }\end{array}$ & $\begin{array}{l}\text { - Grille d'analyse } \\
\text { réflexive et fiches } \\
\text { consignes } \\
\text { - Conduite de } \\
\text { l'enquête réflexive } \\
\text { transverse }\end{array}$ & $\begin{array}{l}\text { Pratique } \\
\text { réflexive } \\
\text { conjointe sur } \\
\text { la conduite du } \\
\text { projet }\end{array}$ & $\begin{array}{l}\text { - Compétences à réfléchir sur } \\
\text { ses propres pratiques } \\
\text { - Compétence d'abstraction et } \\
\text { de généralisation } \\
\text { - Vision partagée des critères } \\
\text { saillants pour la régulation } \\
\text { de l'activité de discussion, } \\
\text { coordination, production }\end{array}$ & $\begin{array}{l}\text { Critères saillants pour la } \\
\text { conduite d'une activité } \\
\text { collaborative } \\
\text { d'organisation des } \\
\text { pratiques }\end{array}$ \\
\hline $\begin{array}{l}\quad 4 \\
\text { Co-construire et } \\
\text { formaliser la } \\
\text { viabilité du } \\
\text { système }\end{array}$ & $\begin{array}{l}\text { - Transfert intentionnel } \\
\text { du pilotage } \\
\text { - Activité de briefing } \\
\text { et débriefing } \\
\text { - Plan de } \\
\text { communication } \\
\text { - Formation des } \\
\text { garants de la } \\
\text { durabilité de la } \\
\text { démarche } \\
\text { - Réunions informelles } \\
\text { (4) } \\
\text { - Réunions de } \\
\text { presentation } \\
\text { opportunistes (5) }\end{array}$ & $\begin{array}{l}\text { Démarche } \\
\text { participative } \\
\text { de production } \\
\text { d'une } \\
\text { nouvelle } \\
\text { prescription }\end{array}$ & $\begin{array}{l}\text { - Management impliqué } \\
\text { - Compétence à manager et } \\
\text { piloter une démarche } \\
\text { collaborative de production } \\
\text { de règles effectives } \\
\text { d'organisation : } \\
\text { - Construire le problème } \\
\text { et impliquer un réseau } \\
\text { d'acteurs pertinents } \\
\text { - Structurer et animer le } \\
\text { débat (activité et REX) } \\
\text { - Organiser et piloter } \\
\text { 1'action collaborative }\end{array}$ & $\begin{array}{l}\text { 1) Procédure de } \\
\text { management d'une } \\
\text { démarche transverse } \\
\text { (règles de production, } \\
\text { coordination et débat) } \\
\text { 2) Procédure REX } \\
\text { Organisationnel (sur les } \\
\text { démarches transverses) } \\
\text { 3) Fiches de } \\
\text { formalisation de l'activité } \\
\text { transverse } \\
\text { 4) Création d'espaces de } \\
\text { discussion production } \\
\text { transverse dans le } \\
\text { fonctionnement quotidien }\end{array}$ \\
\hline
\end{tabular}

\section{Les rôles et la place de l'ergonome}

L'ergonome joue des rôles multiples dans le cadre d'une intervention capacitante. Cette multiplicité est rendue nécessaire et possible par la durée de l'intervention, l'objet de la 
demande, sa visée finale, et son origine. S'interroger sur les différents, voire nouveaux, rôles de l'ergonome amène à s'intéresser aux connaissances qu'il est à même de mobiliser et à questionner les contenus des formations initiales des ergonomes par rapport aux nouveaux défis des interventions capacitantes.

L'intervention décrite ici avait pour objet la conduite d'un projet de redesign des processus organisationnels, où la gouvernance même du changement était objet de transformation. L'intervention visait donc le développement de l'organisation. L'ergonome a donc été engagée, dès l'analyse du système, dans la définition et la réalisation des opportunités de transformation et dans l'ancrage des solutions organisationnelles produites. L'ergonome avait la responsabilité opérationnelle du projet en qualité de "chef d'orchestre» de la démarche. Qu'est-ce que cela a impliqué concrètement pour l'ergonome?

Si l'on examine les productions stratégiques réalisées pour préparer et assurer l'implantation de la démarche, décrites précédemment, les rôles de l'ergonome peuvent être lus sur trois plans : pédagogique, politique et opérationnel. Ceux-ci sont résumés dans le tableau 4 et détaillés dans les sections qui suivent.

Tableau 4 : Récapitulatif des rôles joués par l'ergonome au cours de l'intervention visant le développement.

Table 4: Summary of the roles played by the ergonomist during the developmental intervention

\begin{tabular}{|c|c|c|}
\hline Plan & Rôle & Finalité \\
\hline \multirow[b]{2}{*}{$\begin{array}{l}\text { Plan } \\
\text { opérationnel }\end{array}$} & Chef de projet & $\begin{array}{l}\text { De résultat: outiller et veiller à la cohérence de } \\
\text { l'ensemble de la démarche par rapport aux objectifs } \\
\text { constructifs, productifs et autopoïétiques visés } \\
\text { De processus : créer un environnement-projet } \\
\text { facilitant les dynamiques collaboratives des acteurs- } \\
\text { projet, en tant que levier stratégique pour } \\
\text { l'aboutissement du résultat final }\end{array}$ \\
\hline & $\begin{array}{l}\text { Analyste } \\
\text { et co-concepteur } \\
\text { expert du travail }\end{array}$ & $\begin{array}{l}\text { Apporter la vision de l'activité réelle dans le débat } \\
\text { réflexif avec les autres logiques d'action de } \\
\text { l'entreprise } \\
\text { Intégrer la variabilité inter-fonctions dans la } \\
\text { production et l'expérimentation des scénarii de } \\
\text { prescription } \\
\text { Promouvoir l'intégration du débat réflexif sur le } \\
\text { travail dans le fonctionnement organisationnel en } \\
\text { tant qu'outil de management et de production }\end{array}$ \\
\hline Plan politique & $\begin{array}{l}\text { Conseil } \\
\text { développement } \\
\text { l'organisation }\end{array}$ & $\begin{array}{l}\text { Susciter le besoin } \\
\text { Contribuer à la professionnalisation des strates } \\
\text { hiérarchiques } \\
\text { Intégrer le travail dans la stratégie organisationnelle } \\
\text { à la variable technique comme facteur de } \\
\text { performance }\end{array}$ \\
\hline
\end{tabular}




\begin{tabular}{|c|c|c|}
\hline \multirow{5}{*}{$\begin{array}{l}\text { Plan } \\
\text { pédagogique }\end{array}$} & $\begin{array}{l}\text { Concepteur de } \\
\text { l'ingénierie des espaces } \\
\text { d'apprentissage }\end{array}$ & $\begin{array}{l}\text { Définir les besoins constructifs nécessaires pour } \\
\text { réaliser les productions attendues du projet et } \\
\text { organiser la systématisation conséquente du } \\
\text { processus d'apprentissage }\end{array}$ \\
\hline & $\begin{array}{l}\text { Animateur du travail } \\
\text { collaboratif }\end{array}$ & $\begin{array}{l}\text { Structurer et piloter la séance de travail collaboratif } \\
\text { Assurer la démocratisation de l'espace de parole } \\
\text { Promouvoir une dynamique collaborative transverse } \\
\text { aux strates hiérarchiques et aux différentes fonctions } \\
\text { dans la réalisation de la tâche }\end{array}$ \\
\hline & $\begin{array}{l}\text { Facilitateur de la } \\
\text { réflexion sur le travail }\end{array}$ & $\begin{array}{l}\text { Accompagner l'appropriation de la pratique réflexive } \\
\text { transverse sur le travail en tant qu'outil de } \\
\text { production et de management de l'organisation }\end{array}$ \\
\hline & $\begin{array}{l}\text { Concepteur d'objets } \\
\text { médiateurs } \\
\text { d'apprentissage }\end{array}$ & $\begin{array}{l}\text { Faciliter les apprentissages } \\
\text { Permettre la manipulation et la reconception dans } \\
\text { l'usage }\end{array}$ \\
\hline & Formateur & $\begin{array}{l}\text { Professionnaliser les garants de la durabilité de la } \\
\text { démarche }\end{array}$ \\
\hline
\end{tabular}

\subsection{Le plan opérationnel : l'ergonome comme chef de projet d'une transformation organisationnelle}

Le plan opérationnel renvoie aux productions stratégiques de l'ergonome visant la production de connaissances sur travail et leur opérationnalisation dans la dimension productive de l'intervention. Sur ce plan, au cours de l'intervention, l'ergonome a joué essentiellement deux rôles : celui de chef de projet et celui d'analyste et de co-concepteur expert du travail, garant des conditions d'un processus de construction collective du problème et de la solution.

En tant que chef $d u$ projet d'une transformation organisationnelle, ses actions étaient tournées à la fois vers le résultat et vers le processus.

Concernant le résultat, il veille au respect des engagements pris dans le cahier des charges, en termes de délais, de ressources mobilisées et de productions attendues. Dans cette intervention, le résultat visé renvoyait à la production de solutions (documents, outils, moyens, processus, etc.) permettant de surmonter les contradictions organisationnelles identifiées et d'ancrer des mécanismes d'autopoï̀se organisationnelle: le fonctionnement organisationnel visé devait permettre une dynamique de continuité propre à la vision développementale soutenue. L'action de l'ergonome cherchait à outiller et à veiller à la cohérence de l'ensemble de la démarche par rapport aux objectifs constructifs, productifs et autopoïétiques. Ceci s'est traduit par une reconception dans l'usage des moyens, des structures et des ressources mobilisées faite de manière opportuniste selon les besoins émergents de l'activité.

Concernant le processus, l'ergonome a pris les dynamiques collaboratives entre les acteurs-projet comme objet d'intervention, en tant que levier stratégique pour atteindre 
le résultat final. L'environnement projet (ressources tangibles et immatérielles, structures, processus) mis en place visait à promouvoir un travail collaboratif de production de l'organisation. Dans ce cadre entrent la réalisation du plan de communication, la mise en place de moyens informatiques partagés, le choix de l'architecture de dispositifs pour qu'ils puissent promouvoir un débat transverse et prendre en compte des problématiques réelles et pour qu'ils soient compatibles et intégrés dans le fonctionnement de l'entité. L'ergonome a pris en charge également la mobilisation des compétences pertinentes par rapport à la réalisation du résultat attendu, ainsi que le soutien de leur investissement et engagement dans la démarche.

51 En tant que co-concepteur expert du travail et garant des conditions d'un processus de construction collective du problème et de la solution, l'ergonome a apporté la vision de l'activité réelle dans le débat réflexif avec les autres logiques d'action (inter-fonctions et inter-services) sur des questions de production, de qualité, de sécurité, etc. En particulier, les liens entre les choix organisationnels, les impacts occasionnés sur l'activité collective réelle et in fine sur la performance ont été toujours mis en évidence (cartographiés et débattus) tant en phase d'analyse que de conception de situations futures. En phase d'analyse les différents objets intermédiaires fournis par l'ergonome ont permis de créer un référentiel opératif commun. En phase de conception, l'ergonome a permis la prise en compte de la transversalité des logiques d'action inter-fonctions dans la production et l'expérimentation des scénarii de prescription. Il a ainsi instruit et facilité l'intégration du débat réflexif sur le travail dans le fonctionnement organisationnel en tant qu'outil de management et de production.

\subsection{Le plan politique : l'ergonome en tant que conseiller en développement de l'organisation}

Sur le plan politique, les productions stratégiques de l'ergonome visaient à conseiller les pouvoirs politiques sur différents points :

- sur les éléments du système et leurs interactions concourant à la performance organisationnelle dans toutes ses dimensions (sécurité, qualité, production, etc.);

- sur les opportunités d'optimisation de la performance de l'organisation ;

- sur les approches et les méthodes qui peuvent outiller la stratégie organisationnelle visant le développement de l'organisation. Développement interprété comme résultat d'une interaction indissociable entre santé et performance.

53 L'action de conseil fondée et alimentée par l'analyse du travail visait donc dans un premier temps à susciter le besoin et dans un deuxième temps à contribuer à la fois à la professionnalisation des strates hiérarchiques et à outiller la stratégie managériale pour qu'elle intègre le travail comme facteur de performance.

\subsection{Le plan pédagogique : l'ergonome en tant que concepteur et promoteur de l'ingénierie d'espaces pédagogiques}

Sur le plan pédagogique, les productions stratégiques de l'ergonome visent à accompagner la dimension constructive de la démarche: par exemple, les choix concernant l'ingénierie des espaces d'apprentissage et les actions de médiation visant la réussite dans les apprentissages. Ces stratégies ont été réalisées de manière planifiée ou opportuniste. 

stratégiques concernent la définition des besoins constructifs identifiés lors du diagnostic comme nécessaires pour réaliser les productions attendues du projet, ainsi que la systématisation du processus d'apprentissage. Ce dernier renvoie au choix des méthodes pédagogiques utilisées, des situations d'apprentissages et des situations appréhendées, des modalités de conduite et des outils de médiation des apprentissages en cohérence avec la perspective d'apprentissage dans laquelle l'ergonome s'inscrit. Le LD a mis en place un double mouvement de médiation de l'apprentissage. L'ingénierie visait à supporter les participants dans la résolution de problèmes de plus en plus complexes, que l'état de développement actuel n'aurait pas permis sans accompagnement. Dans ce cadre entrent les actions de l'ergonome de simplification du problème, telles que la modélisation du système de maintenance (cartographie globale) et la mise en place d'un « environnement de projet exemplaire » qui permettait aux participants de s'approprier le modèle d'une démarche participative de l'organisation (en l'expérimentant et en l'enrichissant dans l'usage). L'ingénierie prenait également en compte la planification de la privation progressive de ce même support médiateur (principe de desétayage) jusqu'au transfert complet de la démarche vers les acteurs organisationnels. médiateurs d'apprentissage.

À l'intérieur de chaque situation d'apprentissage (groupe de travail), il était engagé en tant qu'animateur du travail collaboratif. Il structurait la séance : temps, thématiques abordées, répartition des rôles, résultats attendus, consigne pour la situation d'apprentissage, état d'avancement de la démarche. Cela avait pour but de créer un référentiel opératif commun qui concourait à donner du sens à l'implication de chacun dans la situation. Il veillait également à assurer une démocratie de l'espace de parole et une dynamique collaborative transverse aux strates hiérarchiques et aux différentes fonctions dans la réalisation de la tâche, et à fournir les informations ou les aides nécessaires (indication des caractéristiques déterminantes) à la compréhension de la situation d'apprentissage.

61 En tant que facilitateur de la réflexion sur le travail, les productions stratégiques de l'ergonome visaient à accompagner l'appropriation de la pratique réflexive transverse sur le travail, en tant qu'outil de production et de management de l'organisation, amenant à l'autopoïèse organisationnelle. Ce travail d'accompagnement renvoie à la fois à la réification de l'activité réelle pour qu'elle devienne un objet transversalement appréciable, discutable et partagé, ainsi qu'à toute stratégie et technique d'explicitation tournée à la fois vers le passé (analyse rétrospective) et vers le futur (projection vers une 
organisation future). Les différents espaces d'apprentissage ont ainsi été outillés afin de faire expérimenter un double niveau réflexif: sur les pratiques de travail (phase de Travail d'organisation transverse) et sur la manière de réfléchir aux pratiques de travail (phase d'Ancrage des mécanismes autopoïétiques).

À l'intérieur du LD, les apprentissages ont été médiés par des objets dont l'ergonome a été le concepteur. Ces objets visaient à la fois à faciliter la construction des apprentissages nécessaires à la réalisation de chaque étape (cartographies) et à permettre la transition vers l'accomplissement de tâches plus complexes (grille REX). Ces objets devaient être accessibles à tous, permettre de réifier l'activité réelle, permettre l'expérimentation et la reconception participative dans l'usage, devenir des traces organisationnelles permettant l'ancrage de la dynamique réflexive sur le travail dans le fonctionnement quotidien.

$\mathrm{Au}$ cours de l'intervention, l'ergonome a joué également le rôle de formateur des garants de la durabilité de la démarche. Ce dispositif d'apprentissage a été construit (contenu et supports didactiques) sur la base des besoins pédagogiques émergents lors de la phase d'ancrage. En particulier, le contenu portait sur la conduite de démarches de conception participative, sur une approche systémique et centrée sur les facteurs socioorganisationnels et humains, sur la facilitation des dynamiques collaboratives dans les situations de travail.

\section{L'évaluation des résultats d'une intervention développementale}

64 La finalité de cette intervention était de promouvoir à la mise en route de mécanismes autopoḯtiques, permettant aux acteurs de renouveler de manière durable leur organisation sur la base des besoins émergents de l'activité. L'évaluation des résultats d'une intervention demande une analyse réflexive sur les effets induits et leur mise en relation avec l'organisation de la démarche. Dans cette section sera présentée une analyse réflexive sur la stratégie sous-jacente au processus d'évaluation. La mise en récit de ce processus a été organisée autour de deux variables : son objet et sa temporalité.

\subsection{L'objet d'évaluation : l'appropriation des situations d'apprentissage}

Afin de pouvoir évaluer les effets de l'intervention, le processus d'appropriation des situations s'inscrivant dans le LD a été pris comme objet d'analyse réflexive et indicateur de la réussite. L'appropriation de ces situations est interprétée ici comme un des mouvements du développement et comme une partie de ses effets.

66 En particulier, l'évaluation de l'appropriation a reposé à la fois sur l'identification des éléments sur lesquels a porté le processus d'appropriation (résultats) mais également sur la mise en relation de ceux-ci avec les conditions par lesquelles l'appropriation a été facilitée, pilotée et promue (processus).

Dans le premier cas, on a examiné les productions matérielles et immatérielles rendues possibles par la médiation des différentes espaces d'apprentissage. Les productions matérielles, résultats de la dimension productive de la démarche, concernent les solutions résultant des activités où les acteurs étaient engagés à chaque étape, c'est-à-dire les livrables. Les productions réalisées à une étape représentaient les données d'entrée 
pour l'étape suivante. Les productions intangibles renvoient à la dimension constructive de la démarche. Elles font référence aux savoirs implicites et actionnables, aux compétences construites par la médiation de l'ingénierie des espaces d'apprentissages mise en œuvre. Ces productions représentent les ressources nécessaires à la fois à la réalisation de chaque étape et aussi à la mise en mouvement de l'autopoï̀se organisationnelle.

Les conditions permettant l'appropriation renvoient à la fois aux choix d'agencement des situations d'apprentissage (le contenu de la tâche et les moyens de réalisation mis à disposition) et aux actions stratégiques, planifiées ou opportunistes, mises en place par l'ergonome pour en faciliter la réussite.

\subsection{La temporalité de l'évaluation : une analyse réflexive tout au long de la démarche}

La mise en route effective des mécanismes autopoiétiques dépend de l'efficacité du rôle médiateur de l'environnement (Laboratoire Développemental) à l'intérieur duquel sont construites les ressources nécessaires. La structuration et la planification des étapes s'inscrivant dans cet environnement ont été réajustées de manière opportuniste au long de la démarche. L'évaluation continue représente donc un facteur de réussite de la démarche.

Ce processus a pris la forme d'une pratique réflexive conduite à la fois en situation (diagnostic) et a posteriori par le biais d'une confrontation de l'ergonome aux enregistrements des situations d'apprentissage qu'il avait mises en place.

71 La réflexion portait sur la mise à l'épreuve des hypothèses initiales sous-jacentes à la conduite et la structuration de chaque dispositif (scénario de prescription de l'ergonome) face au déroulement des situations réelles. L'analyse consistait en mettre en relation les objectifs initiaux (que veux-je obtenir?), les modalités de conduite de la démarche (par quel moyen ?) et les effets réellement engendrés (de quelle nature : positive ou négative? ). Cette première étape réflexive ouvrait sur un questionnement ultérieur sur les leviers de l'efficacité, sur les facteurs de blocage, sur les actions de réajustement à prévoir et les éléments à généraliser pour le futur.

72 Ce processus d'évaluation a permis à la fois de répondre aux besoins constructifs et productifs émergeant lors de chaque situation et d'assurer la pertinence de la démarche. Mais aussi de capitaliser des apprentissages pour le développement des pratiques d'intervention ergonomique.

\subsection{Synthèse des résultats de l'intervention}

L'évaluation de l'apprentissage a été réalisée sur la base de trois catégories d'indices :

- la résolution même du problème contenu dans la situation d'apprentissage, dans sa dimension matérielle (production des cartographies, plan d'actions, référentiels procéduraux) et processuelle (réalisation d'un diagnostic global, d'une analyse détaillée, d'une analyse réflexive sur les pratiques de conduite, etc.) ;

- l'émergence de nouveaux concepts (compétences d'auto-enquête, formation d'un référentiel opératif commun, etc.) ; 
- l'opérationnalisation de manière autonome (non demandée par l'ergonome) des savoirs acquis dans d'autres situations.

l'ergonome ont permis aux participants de réaliser une analyse réflexive sur les pratiques
de conduite du projet de redesign des processus. généralisation de leurs propres pratiques managériales et ont abouti à une vision partagée des éléments clés pour la conduite d'une démarche transverse. Cela a été mis en évidence par la généralisation de la réflexion de la conduite du projet aux pratiques managériales en vigueur. Ce travail a permis d'aboutir à la production d'un certain nombre de critères de qualité pour la conduite d'une démarche collaborative d'organisation des pratiques.

79 Lors de l'étape de «co-construction et formalisation de la viabilité du système autopoḯtique » (cf. Tableau 3, étape 4), l'ergonome a transféré intentionnellement le management de la démarche aux acteurs organisationnels. Néanmoins il a soutenu ce transfert par une activité de briefing et débriefing planifié et opportuniste, par une formation dédiée aux acteurs garants de la durabilité de la démarche, par des réunions opportunistes de présentation de la démarche aux nouveaux agents. La réalisation même de cette étape représente un indice de réussite de la mise en mouvement des mécanismes autopoiétiques. Les acteurs ont su réaliser une démarche de conception participative d'une nouvelle prescription concernant les pratiques de construction d'un problème, de définition d'un réseau d'acteurs pertinents, de structuration et d'animation du débat sur le travail, de coordination et pilotage de l'action collaborative. L'ensemble de la démarche 
a été fondé sur une analyse des besoins de l'activité réelle et les scénarii de prescription ont été mis à l'épreuve dans les pratiques réelles.

Cette étape a abouti à la réalisation d'un référentiel de management d'une démarche transverse, d'un référentiel de REX organisationnel (sur les démarches transverses) et de fiches de formalisation de l'activité transverse. En parallèle de la démarche de production de la nouvelle prescription, huit espaces de débat réflexif sur les pratiques opportunistes ont été lancés et pilotés par des agents de maîtrise.

Des indices d'usage ont été remontés 4 mois après le départ de l'ergonome. En particulier, l'ensemble des référentiels produits a été intégré dans le système de gestion des procédures RATP. L'utilisation de ces référentiels a été étendue à la gestion d'incidents nécessitant la coordination de plusieurs acteurs externes. Le contenu de la formation aux garants de la durabilité a été décliné par l'animateur qualité aux agents de maitrise censés prendre en charge le pilotage de futures démarches transverses. Quatre démarches participatives ont été lancées et pilotées à l'aide des référentiels produits. Finalement, l'ensemble de la démarche a fait l'objet d'une présentation de la part du chef d'entité au niveau central afin de partager le retour d'expérience avec d'autres entités.

\section{Discussion}

Dans cette section seront discutés certains éléments de contribution à la réflexion sur les interventions capacitantes. L'invitation à la réflexion est adressée à la communauté des ergonomes, mais aussi à tous les experts du travail censés intervenir sur des transformations organisationnelles.

\subsection{Quels leviers pour une intervention capacitante et durable ?}

Dans cette intervention le développement a été recherché par la mise en place de mécanismes autopoïétiques permettant à l'organisation de se renouveler de manière autonome selon les besoins émergents de l'activité et de son environnement. Trois facteurs ont contribué à la réussite de la démarche.

Le premier consiste à fonder stratégiquement et politiquement la démarche. Il s'agit de développer la compétence managériale à l'intégration dans la stratégie d'entreprise des questions socio-organisationnelles et humaines. Ceci passe par une activité de conseil au développement organisationnel et par la participation au niveau opérationnel des différentes strates hiérarchiques.

Le deuxième porte sur la systématisation du processus d'apprentissage. Il s'agit de traduire les éléments issus du diagnostic en besoins d'apprentissage nécessaires à la réalisation des productions attendues. En particulier, le Laboratoire développemental (LD) a été conçu comme un environnement exemplaire visant à permettre aux acteurs organisationnels de construire les apprentissages nécessaires en faisant l'expérience et en mettant à l'épreuve de nouvelles manières d'organiser les pratiques au cours même de ces productions. Le LD a donc une double orientation. La première de nature pédagogique, constructive, orientée vers les acteurs et vers l'organisation-processus. Le LD fonctionne comme un dispositif pédagogique de type ZPD, où les acteurs sont mis en situation d'apprentissage (assisté) par la pratique. La deuxième de nature fonctionnelle, productive, orientée vers l'organisation-artefact. Le LD fonctionne comme un «FabLab», à l'intérieur duquel les 
acteurs organisationnels inventent et testent de nouvelles formes et mécanismes organisationnels. Chacune de ces orientations alimente l'autre.

Le troisième concerne l'ancrage des dispositifs d'intervention dans le fonctionnement organisationnel. Le Laboratoire développemental a représenté un lieu d'apprentissage au cours desquels les moyens pédagogiques étaient transformés dans l'usage en outils de management. Un facteur ultérieur facilitant l'ancrage est le réagencement des ressources présentes dans l'entreprise afin qu'elles puissent répondre aux besoins constructifs, productifs et autopoïétiques visés. L'identification et la mobilisation d'acteurs garants de la durabilité de la démarche facilitent également l'ancrage dans le fonctionnement organisationnel.

\subsection{Quelles interrogations pour la formation initiale des ergonomes?}

La multiplicité des rôles joués par l'ergonome a représenté une des conditions qui ont concouru à tenir ensemble les différents objectifs et à assurer la réussite de la démarche. Réfléchir aux différents rôles que l'ergonome a joués permet de se focaliser sur certaines compétences clés émergentes qui peuvent questionner la formation initiale des jeunes ergonomes.

On discutera donc ici plus particulièrement les rôles aux plans politique et pédagogique.

Sur le plan politique, l'ergonome a été engagé dans une activité de conseil au développement organisationnel. C'est à ce niveau qui se jouent les marges de manœuvre pour l'ergonome, qui permet d'ouvrir sur des possibilités d'intervention à plus large spectre par rapport à une mission courte. Et même dans ce dernier cas, c'est au niveau politique que sont négociées les marges de décision et d'action de l'ergonome concernant le pilotage et la structuration de la démarche.

Intervenir au niveau politique demande un investissement important de la part de l'ergonome dans la construction d'un « discours » qui soit acceptable et fonctionnel pour les décideurs hiérarchiques. Cela a différentes implications. En premier lieu, l'identification de l'enjeu principal de l'entreprise (dans l'intervention présentée, c'était la performance déclinée dans ses dimensions de sécurité, de continuité de service et de production). Sur la base de cet élément, l'ergonome doit développer des stratégies et des méthodes de démonstration des liens de causes à effets entre choix organisationnels et enjeu principal pour l'entreprise. Le but de cette démonstration est double: faire apprécier le champ d'application de l'ergonomie (souvent limité aux aspects physiques et de santé) et montrer l'utilité d'une approche systémique au développement de la performance. Le discours ainsi construit nécessite d'être alimenté et renégocié tout au long de la démarche. Mais, au-delà de ce discours démonstratif, l'ergonome doit également mobiliser et soutenir l'engagement des décideurs dans les groupes opérationnels pour assurer une controverse transverse (aux fonctions et aux niveaux hiérarchiques) sur le travail. Ces décideurs sont les légitimateurs et les garants de la durabilité de la démarche.

91 L'ensemble de ces actions demande des compétences de négociation, de traduction du discours ergonomique en langage "politique-stratégique", mais également de «marketing » de l'ergonomie. Ces aspects, trop peu présents dans les formations initiales à l'ergonomie, mériteraient d'être mieux pris en compte dans les programmes. 
Sur le plan pédagogique, nous avons souligné que l'apprentissage représente le moyen pour atteindre le développement de l'organisation, des activités et des collectifs. Cet élément n'est pas disjoint de la dimension productive et contextuelle de la démarche. Dans ce cadre, l'ergonome est appelé à anticiper différents éléments: les besoins d'apprentissage nécessaires à réaliser les solutions productives attendues à chaque phase du projet, l'ordre d'enchainement des situations d'apprentissage, les objets médiateurs mieux à même de faciliter le processus d'acquisition, les actions de maintien de la cohérence de la démarche globale par rapport aux intentions de transfert. L'anticipation de ces éléments n'est possible qu'à partir des éléments issus du diagnostic, initial ou en situation au cours de la démarche. C'est l'analyse continue des situations s'inscrivant dans les dispositifs d'intervention qui permettent à l'ergonome d'identifier les dysfonctionnements, les freins, les leviers et les opportunités. Finalement c'est l'analyse de l'activité réalisée hic et nunc qui permet à l'ergonome d'acquérir une intelligence des situations en objet et de les ré-agencer pour répondre aux objectifs à la fois constructifs, productifs et autopoḯtiques et enfin d'assurer la réussite de l'intervention. Ces éléments informent sur l'état de développement de l'organisation et permettent à l'ergonome d'ouvrir sur les opportunités de transformation. C'est à partir de ces dernières que l'ergonome anticipe et planifie l'ingénierie d'intervention, qui sera réajustée au fur et à mesure sur la base des besoins émergents. Au-delà de l'activité de conception des espaces d'apprentissage, l'ergonome est également engagé dans la gestion des dynamiques collectives d'apprentissage. Dynamiques qui renvoient non seulement au contenu mais aussi à la gestion de situations collectives d'apprentissage. Ces dernières concernent la mise en œuvre de techniques d'apprentissage participatif et la gestion des conflits qui peuvent s'instaurer entre les participants ou avec l'ergonome (actions de boycott, postures de participation "passive», etc.). Tous ces éléments dépassent la seule formation professionnelle des acteurs organisationnels. Ils s'intègrent dans la stratégie ergonomique d'accompagnement d'une transformation de l'organisation. Ils jouent donc un rôle clé dans la réussite d'une démarche capacitante visant le développement dans une visée durable.

Actuellement les formations initiales à l'ergonomie sont plutôt orientées vers la dimension productive. Les programmes fournissent les outils d'analyse du travail, pour créer et piloter les espaces de conception, pour produire et faire produire des objets d'aide à la réification de l'activité, à la simulation de l'activité et d'aide à la conception participative. Les dimensions constructives et organisationnelles restent encore peu explorées et opérationnalisées et constituent donc un axe d'investissement pour le développement des pratiques d'intervention.

\section{BIBLIOGRAPHIE}

Arnoud, J., \& Falzon, P. (2017). Intervenir « sur » l'organisation : vers un cadre méthodologique. In A.-L. Ulmann, A. Weill-Fassina, \& H. Benchekroun (Eds.), Intervenir - Histoires, recherches, pratiques. Toulouse : Octarès. 
Barcellini, F. (2015). Développer des interventions capacitantes en conduite du changement. Comprendre le travail collectif de conception, agir sur la conception collective du travail. Habilitation à Diriger des Recherches, Université de Bordeaux, Bordeaux.

Barcellini, F., Van Belleghem, L., \& Daniellou, F. (2013). Les projets de conception comme opportunité de développements des activités. In P. Falzon (Ed), Ergonomie constructive (pp. 191-206). Paris: PUF.

Boreham, N. (2004). A theory of collective competence: challengin the Neo-Liberal Individualisation of Performance at work. British Journal of Educational Studies, 52(1), 5-17.

Broberg, O., Andersen, V., \& Seim, R. (2011). Participatory ergonomics in design processes: The role of boundary objects. Applied Ergonomics, 42, 464-472.

Bruner, J. S. (1983). Le développement de l'enfant : savoir faire, savoir dire. Paris : PUF.

Carta, G., Falzon, P., \& Re, A. (2015). A constructive and participative approach to the analysis and redesign of the organization. 19th Triennial Congress of the International Ergonomics Association. Melbourne, 9-14 August 2015.

Casse, C. (2015). Concevoir un dispositif de retour d'expérience intégrant l'activité réflexive collective : un enjeu de sécurité dans les tunnels routiers. Thèse de doctorat. Université Grenoble Alpes.

Chia, R. (2002). Essai: Time, duration and simultaneity: Rethinking process and change in organizational analysis. Organization Studies, 23(6), 863-868.

Dugué, B., Petit, J., \& Daniellou, F. (2010). L'intervention ergonomique comme acte pédagogique. Pistes, 12(3).

Dewey, J. (1938). Logic. The theory of Inquiry. New York : Henry Holt \& Co [en français : John Dewey, Logique (La théorie de l'enquête), Paris : PUF, 2006].

Engeström, Y., Sannino, A., \& Virkkunen, J. (2014). On the methodological demands of formative interventions. Mind, Culture, and Activitiy, 21(2), 118-128.

Falzon, P. (2005). Ergonomie, conception et développement. Conférence introductive, 40ème Congrès de la SELF, Saint-Denis, La Réunion, 21-23 septembre 2005.

Falzon, P. (2013). Pour une ergonomie constructive. In P. Falzon (Ed), Ergonomie constructive (pp. 1-15). Paris : PUF.

Falzon, P., \& Barcellini, F. (2014). Former à la pratique en ergonomie- Texte de cadrage relatif à l'accompagnement du mémoire professionnel d'ergonomie. Cours «ERG 245 : Intervenir en ergonomie » du CNAM, octobre 2014.

Feldman, M. S. (2000). Organizational routines as a source of continuous change. Organization Science, 11(6), 611-629.

Gherardi, S. (2000). Practice-based theorizing on learning and knowing in organizations: An introduction. Organization, 7(2), 211-223.

Hernes, T. (2008). Understanding organization as process. Theory for a tangled world. New York: Routledge.

Hernes, T. (2010). Actor-network theory, Callon's scallops, and process-based organization studies. In T. Hernes \& S. Maitlis (Eds.), Process, sensemaking and organizing (pp. 161-184). Oxford: Oxford University Press. 
Hernes, T., \& Maitlis, S. (2010). Process, sensemaking and organizing: An introduction. In T. Hernes \& S. Maitlis (Eds.), Process, sensemaking and organizing (pp. 27-37). Oxford : Oxford University Press.

Lorino, P. (2009). Concevoir l'activité collective conjointe : l'enquête dialogique. Étude de cas sur la sécurité dans l'industrie du bâtiment. Activités, 6(1), 87-110. URL : https:// activites.revues.org/2154

Lorino, P. (2013). L'activité collective, processus organisant. Un processus discursif fondé sur le langage pragmatiste des habitudes. Activités, 10(1), 221-242. URL : https://activites.revues.org/656 Lorino, P., Tricard, B., \& Clot, Y. (2011). Research methods for nonrepresentational approaches to organizational complexity: The dialogical mediated inquiry. Organization Studies, 32(6), 769-801.

Lorino, P., \& Mourey, D. (2013). The experience of time in the inter-organizing inquiry: A present thickened by dialog and situations. Scandinavian Journal of Management, 29, 48-62.

Mayen, P. (2012), L'appropriation des situations. In Clot (Ed.), Vigotski maintenant (pp. 289-305). Paris : La Dispute.

Maturana, H., \& Varela, F. (1980). Autopoiesis: the organisation of the living. In H. Maturana, \& F. Varela (Eds.), Autopoiesis and Cognition: The Realisation of the Living. Dordecht: D. Reidel Publishing.

Nicolini, D., Gherardi, S., \& Yanow, D. (2003). (Eds.) Knowing in organizations: A practice-based approach. Armonk, NY : M. E. Sharpe.

Noyer, N., \& Barcellini, F. (2014). Construire un projet durable de prévention des TMS : dimensions stratégique et pédagogique d'une intervention ergonomique. Pistes, 16(3).

Orlikowski, W. J. (2000). Using technology and constructing structures: A practice lens for studying technology in organizations. Organization Science, 11(4), 404-428.

Orlikowski, W. J. (2007). Sociomaterial practices: Exploring technology at work. Organization Studies, 28, 1435-1448.

Pastré, P. (2005). Apprendre par la simulation : de l'analyse du travail aux apprentissages professionnels. Toulouse, France : Octarès.

Petit, J. (2005). Organiser la continuité du service : intervention sur l'organisation d'une Mutuelle de santé . Thèse de doctorat en ergonomie. Bordeaux : Éditions du Laboratoire d'ergonomie des systèmes complexes.

Petit, J. (2006). Experimentation as means of intervention for the ergonomist in the management of an organizational change? À case study. In IEA 2006, XVIth World Congress on Ergonomics. Maastricht, Pays-Bas.

Quick, T. (2003). 'Autopoiesis', available at : www.cs.ucl.ac.uk/staff/t.quick/autopoiesis.html Raspaud, A. (2014). De la compréhension collective de l'activité réelle à la conception participative de l'organisation : plaidoyer pour une intervention ergonomique capacitante. Thèse de doctorat en ergonomie, Conservatoire National des Arts et Métiers-CNAM.

Ricoeur, P. (1984). Temps et récit, tome I : L’intrigue et le récit philosophique. Paris: Le Seuil.

Rocha, R., Mollo, V., \& Daniellou, F., (2015). Work debate spaces: A tool for developing a participatory safety. Applied Ergonomics, 46, 107-114.

Samurcay R., \& Rabardel P. (2004). Modèles pour l'analyse de l'activité et des compétences : proposition. In R. Samurçay \& P. Pastré (Eds.), Recherches en didactique professionnelle (pp. 163-180). Toulouse : Octarès. 
Seim, R., Broberg, O., \& Andersen, V. (2014) Ergonomics in Design Processes: The Journey from Ergonomist toward Workspace Designer. Human Factors and Ergonomics in Manufacturing, 24, 650-670.

Schön, D. (1983). The reflective practitioner: how professionals think in action. New York: Basic Books.

St-Vincent, M., Vézina, N., Bellemare, M., Denys, D., Ledoux, E., \& Imbeau, D. (2011). L'intervention en ergonomie. Québec: Éditions MultiMondes, IRSST.

Tsoukas, H. (2009). A dialogical approach to the creation of new knowledge in organizations. Organization Science, 20(6), 941-957.

Tsoukas, H., \& Chia, R. (2002). On organizational becoming: Rethinking organizational change. Organization Science, 13(5), 567-582.

Varela, F. (1989). Autonomie et connaissance. Essai sur le vivant, Paris, Le Seuil, coll. « La couleur des idées ».

Varela, F.G., Maturana, H.R., \& Uribea, R. (1974). Autopoiesis: The organization of living systems, its characterization and a model. Biosystems, 5(4), 187-196.

Vygotsky, L.S. (1930/1983). Immaginazione e creatività nell'età infantile. Paideia, Italy : Editori Riuniti.

Vygotski., L. (1997). Pensée et Langage. Paris : Éditions La Dispute.

Weick, K. E. (1977). Organisation design: Organisations as self-designing systems. Organisational Dynamics, 6(2): 30-46

Weick, K. E. (1995). Sensemaking in Organizations. Thousands Oaks, CA: Sage.

Weick, K. E. (2012). Making sense of the organization: Volume 2: The impermanent.

Weick, K. E., Sutcliffe, K.M., \& Obstfeld, D. (2005). Organizing and process of sensemaking. Organization Science, 16(4), 409-421.

Wilson, J. R. (2012). Fundamentals of systems ergonomics. Work, 41, 3861-3868.

\section{RÉSUMÉS}

Ce papier présente une intervention ergonomique portant sur la reconception participative des processus organisationnels dans le milieu ferroviaire. L'intervention est déclenchée suite à l'internalisation partielle de l'activité de maintenance de la signalisation du métro. La conduite davantage techno-centrée de ce changement a impacté la fiabilité et la performance organisationnelle. L'intervention misait le développement de l'organisation dans une visée durable. L'ingénierie des dispositifs d'intervention a été conçue comme un «Laboratoire Développemental ». Ce dernier vise à outiller les acteurs organisationnels dans la construction et la mise en marche de mécanismes autopoïétiques, autrement dit, qui permettent à l'organisation de se renouveler de manière autonome selon les besoins émergents de l'activité. Ainsi, la dimension constructive de l'activité est prise comme levier pour le développement de l'organisation. L'analyse réflexive a posteriori de cette expérience vise à mettre en évidence les conditions, les stratégies et la multiplicité des rôles que l'ergonome peut jouer en tant que manager de la transformation organisationnelle. 
This paper describes a participatory intervention aimed at redesigning an organization in the railway sector. The intervention took place after an attempt at insourcing part of the subway maintenance activity. The techno-centered management of the change resulted in a decrease in organizational reliability and performance. The intervention focused on the sustainability of the organizational development. The entire intervention was conceived as a "Developmental Laboratory" aimed at empowering organizational actors to construct and set in motion autopoietic mechanisms, i.e. mechanisms allowing actors to permanently redesign the organization to suit activity requirements. The constructive dimension of the activity is taken as a lever for organizational development. A post-hoc reflexive analysis reveals the conditions of such a developmental intervention strategy and the multiple roles played by the ergonomist as an organizational change manager.

\section{INDEX}

Keywords : enabling intervention, developmental laboratory, organizational autopoiesis, participatory redesign or processes

Mots-clés : intervention capacitante, laboratoire développemental, autopoïèse organisationnelle, reconception participative des processus

\section{AUTEURS}

\section{GIANNA CARTA}

Conservatoire National des Arts et Métiers (CNAM), CRTD, 75005 Paris.

\section{PIERRE FALZON}

Conservatoire National des Arts et Métiers (CNAM), CRTD, 75005 Paris. pierre.falzon@cnam.fr 